\title{
LES MÉDIA-ACTEURS DE PROXIMITÉ : DES CITOYENS EN QUÊTE DE COMMUN
}

\begin{abstract}
Alain Bouldoires et Christine Larrazet ${ }^{1}$
Cet article rend compte des résultats d'une enquête empirique, la «Caravane des médias d'initiative populaire », centrée sur la prise de parole dans l'espace public de citoyens ayant créé, à leur seule initiative, un espace de communication et de délibération sur internet (webradio, webTV, webmagazine). Les entretiens avec ces sujets média-actifs, ici nommés média-acteurs de proximité, dressent l'esquisse d'individus en prise directe avec leur territoire local qui cherchent à concilier parole individuelle et action collective. Ces média-acteurs de proximité se saisissent de l'arène démocratique locale, rurale ou urbaine, comme un commun qu'ils estiment pouvoir et devoir investir, aux côtés d'autres paroles dominantes, qu'elles soient institutionnelles ou professionnelles. Mettant en pratique la vita activa promue par Hannah Arendt, ces citoyens parviennent à articuler l'espace informationnel virtuel avec la matérialité du monde réel.
\end{abstract}

Par bien des aspects, les notions de « monde commun » ou de « bien public » apparaissent comme un « trésor perdu (Tassin, 1999).

1 Alain Bouldoires est Maître de conférences à l'Université Bordeaux Montaigne - Laboratoire MICA est rédacteur en chef de la Revue Française des Méthodes Visuelles Christine Larrazet est américaniste, Maître de conférences au Collège des Sciences de l'Homme de l'Université de Bordeaux, et chercheuse au Centre Emile Durkheim (UMR CNRS 5116).

Recherches en communication, $\mathrm{n}^{\circ} 42$ (2016). 
De multiples facteurs ont contribué à la dissolution de notre rapport au monde moderne : les excès de la consommation, du chômage, de la croissance ou des technosciences ont été autant de forces destructrices des libertés et du « bonheur public ». Les intérêts privés ont ainsi peu à peu pris la place du politique, du partage, de la dignité humaine. Le concept de « capital social », développé par les travaux de Pierre Bourdieu en tant que réseaux de relations, puis par le sociologue américain Robert $D$. Putnam ${ }^{1}$ dans une acception légèrement différente, permet de penser une alternative à une vision purement utilitariste de la société : la vitalité des réseaux d'interrelations travaillerait le lien social. Pour Bernard Perret, ce « capital social » ne suffirait cependant pas à assurer la cohésion de la société et l'auteur propose de combiner un « agir instrumental » des individus avec un « agir constitutif» qui s'inscrive dans une totalité sociale (Perret, 2003). Ainsi, même s'il est nécessaire de penser la construction symbolique du monde dans lequel les individus prennent place, la question de l'action individuelle reste posée. En effet la réflexion sur le «capital social », la démocratie et le « monde commun », butte sans cesse sur la question du comment : Comment prendre part activement et individuellement à la démocratie?

Pour Hannah Arendt le vivre ensemble est essentiellement un espace de liberté et d'échange où peut s'inscrire l'action politique. Elle propose, pour l'accomplir, le concept de vita activa qui fait de l'humain, dans toute sa pluralité, un acteur du monde commun (Arendt, 2012). La vita activa selon Arendt implique trois activités humaines fondamentales : le travail (qui maintient le processus vital), l'œuvre (la création d'objets) et l'action, étroitement apparentée à la parole, qui serait « la seule activité qui mette directement en rapport les hommes » (Arendt, 2012, p. 65). Arendt place ainsi l'échange de paroles au cœur de sa conception de la vie politique associée à la reconnaissance de la pluralité qui serait, selon elle, la condition per quam de toute vie politique.

1 Ce concept de capital social, initié en 1988 dans un article intitulé « Social Capital in the Creation of Human Capital» (American Journal of Sociology, volume 94) par le sociologue James Coleman, a été repris par Robert D. Putnam dans un long article intitulé « Bowling Alone: America's Declining Social Capital » (Journal of Democracy, janvier 1995) qui a donné lieu à un livre paru aux Etats-Unis en 2000 sous le titre Bowling Alone. The Collapse and Revival of American Community. Le politologue américain y décrit le déclin de la vie sociale aux Etats-Unis et ses conséquences néfastes sur l'action collective et la démocratie. 
Pour les classes populaires, à défaut d'un capital culturel au sens de Bourdieu, le fait d'être d'un lieu, de participer d'une société d'interconnaissance est une ressource pour l'action. Ainsi diverses pratiques collectives, parfois anciennes, parfois nouvelles, contribuent à mettre en échec la privatisation systématique de la vie humaine. Le « commun » à instituer est donc moins juridique ou étatique que sociétal. Il repose sur une dynamique « par le bas » d'investissement de l'espace public à inscrire dans le temps.

$\mathrm{Au}$ début du XXIe siècle, grâce à sa plus grande accessibilité, Internet semble pouvoir être l'outil qui permettrait l'échange de paroles plurielles, célébré par Hannah Arendt, dans un nouvel espace public. L'espace public numérique, vécu comme une agora élargie où traiter de la chose publique, est aujourd'hui investi d'une pluralité de points de vue. Depuis deux décennies à peine, la prise de parole citoyenne, par des citoyens ordinaires, ni journalistes, ni politiques, ni écrivains, ni pamphlétaires, est rendue possible par Internet. Mais alors que de nombreux travaux ont exploré l'impact d'Internet et de son accessibilité sur la démocratie, peu d'études empiriques sont allés à la rencontre des citoyens qui investissent cet espace de leur action individuelle hors des médias professionnels. Quelles intentions animent ces prises de paroles à la marge? Cherchent-elles véritablement à concilier parole individuelle et action collective ? Comment s'expriment-elles et avec quel idéal ?

Cet article tente de répondre à ces questions, en rendant compte des résultats d'une enquête empirique, la « Caravane des médias d'initiative populaire », centrée sur la prise de parole de citoyens ayant créé un espace de communication et de délibération sur Internet (webradio, webTV, webmagazine) à leur seule initiative et sans soutien de professionnels du journalisme, ce qui les distingue du « Journalisme Participatif et Citoyen sur Internet » $(\mathrm{JPCI})^{1}$ et de la prise de parole citoyenne insérée dans les médias (Aubert, 2009). Cette recherche étudie le processus de la construction de la citoyenneté, à l'ère du numérique, et analyse les conditions de l'expression publique dans l'espace démocratique. Les entretiens avec ces citoyens, identifiés et rencontrés au fil de

1 Le terme «média citoyen » est parfois employé pour désigner une collaboration entre journalistes professionnels et des citoyens non journalistes (Cardon, Granjon, 2010, p.116). Pour éviter une confusion avec la définition qui prévaut à l'échelle internationale, celle de Clemencia Rodriguez, « citizen media » (Rodriguez, 2001), nous préférons le terme de « journalisme participatif et citoyen sur internet » (JPCI) pour référer à cette collaboration (Pelissier, Chaudy, 2009). 
l'enquête, dessinent des individus « à la fois individuels et collectifs » (Badiou \& Gaucher, 2014), qui se saisissent de l'arène démocratique locale, rurale ou urbaine, comme d'un commun qu'ils estiment pouvoir et devoir investir, aux côtés d'autres paroles dominantes, qu'elles soient institutionnelles ou professionnelles. Ils y mènent la vita activa célébrée par Hannah Arendt.

\section{Les expérimentations médiatiques citoyennes}

Comme l'a montré la sociologie de la communication, l'espace public est dominé par les mass-médias (Habermas, 1992). Parmi eux, les médias d'information, longtemps considérés comme des relais à la parole citoyenne et comme un contrepouvoir nécessaire à la démocratie, ont beaucoup perdu en représentativité alors qu'Internet se présente aujourd'hui comme un outil permettant d'ouvrir un espace public plus large (Gingras, 2009 ; Cardon \& Granjon, 2010). Internet, comme espace de diffusion de voix singulières, dégagerait un autre ou d'autres espaces communs de discussion que l'espace public unique identifié par Habermas dans son étude historique de la politique britannique (Habermas, 1988). Un espace unique que de nombreux chercheurs, et Habermas lui-même, ont élargi à des espaces publics au pluriel occupés par plusieurs publics et contrepublics (Habermas, 1992; Fraser, 1990 ; Calhoun, 2010). Hors du contrôle qui préside à la construction du discours des médias d'information, l'espace public numérique apparait aujourd'hui comme un commun que chacun peut investir sans intermédiaire et où se mènent des expérimentations citoyennes plurielles.

Les technologies de l'information et de la communication et la société se transforment mutuellement comme le montrent les études sur les publics et les usages des différents médias depuis les années 1950. Le thème de la sociabilité, en particulier, a fait l'objet de nombreuses contributions ${ }^{1}$. Une des thèses avancées par certains chercheurs ${ }^{2}$ est l'hypothèse continuiste : les outils de communication étendent

1 Pour un regard rétrospectif sur ces questions, nous renvoyons à l'excellent numéro anniversaire de la revue Réseaux $\left(\mathrm{n}^{\circ} 184-185,2014\right)$ qui fête 30 ans de publications sur le lien entre technologie, communication et société. Les auteurs cités dans ce paragraphe proviennent des synthèses réalisées dans ce numéro double. https:// www.msha.fr/citoyenneteencouleurs/

2 Claisse, Vergnaud, Chabrol, Perin, Rivière, Smoreda, Licoppe, Lambiotte, Singly, Bidart. 
la sociabilité des individus. Il y a continuité entre la «sociabilité en face-à-face et celle médiatisée par les outils de communication» (Cardon, Smoreda, 2014, p. 166). D'autres contributions soulignent l'entrelacement et la reconfiguration des formats de l'échange1. Selon Dominique Cardon et Zbigniew Smorera, «certes, les liens forts des individus se renforcent sans se transformer fondamentalement avec l'usage des outils de communication, en revanche, les nouvelles technologies relationnelles ouvrent un espace nouveau et original pour organiser et interagir avec les liens plus faibles» (Cardon, Smoreda, 2014, p.163). Mais la force des «liens faibles» (amis Facebook, collègues LinkedIn, mais aussi les internautes des «médias d'initiative populaire»), activés par les moyens numériques, réside essentiellement dans le caractère instrumental : le dispositif de communication permet avant tout de prolonger l'action ${ }^{2}$. Ainsi, des collectifs en ligne, moteurs de sociabilités, se forment avec une intensité du travail d'interaction souligné par de nombreux auteurs ${ }^{3}$. Dans la réalité des pratiques relationnelles des internautes, les études en témoignent (Grossetti, 2014), les liens faibles ne sont pas, la plupart du temps, associés à des relations artificielles ou opportunistes.

Les récentes études menées sur les expérimentations citoyennes dans l'espace public numérique ont contribué tout autant à identifier les nouveaux espaces publics pluriels, qu'à documenter l'impact de l'usage des outils de communication numérique sur les liens sociaux. Tout en participant à faire la « cartographie des sites de débat public », pour reprendre un terme de François et Neveu (François \& Neveu, 1999), elles ont cherché en particulier à saisir ce qui anime le phénomène participatif. Tout un pan de la recherche révèle un ancrage militant issu de mobilisations collectives. Dans cette sphère publique alternative, se trouvent des intentions multiples susceptibles de s'entrecroiser : comme l'opposition aux médias d'information mainstream, nourrie d'une visée anti-hégémonique ou expressiviste (Cardon \& Granjon, 2010), ou encore la diffusion de points de vue diversifiés, s'appuyant sur une visée émancipatrice et d'empowerment (Rodriguez, 2001). Les ressorts de l'action individuelle ont été observés dans un autre pan des

1 Cardon, Rainie, Wellman, Beaudouin, Velkovska, Licoppe, Smoreda.

2 Cette réticence a été également révélée dans une autre enquête du programme Citoyennetés en couleur sur le Slam. Voir Alain Bouldoires, Christine Larrazet, «Espace libre pour un chercheur slameur : De l'observation à la traversée de l'objectif », Enquêtes et Ancrages, n¹, 2017. http://revue-ancrages.fr/.

3 Licoppe, Beaudouin, Cardon. 
recherches centrées sur l'expression citoyenne adossée aux médias professionnels (Gillmor, 2006; Aubert, 2014). Dans ce champ du journalisme participatif, ou de la coopération « pro-am », les études ont cherché à démêler ce qui relèverait de l'idéal démocratique, de l'engagement, ou de la production de soi. Pour Gilmor, «l'activisme informationnel » (news activism) témoigne d'un plus grand engagement des citoyens dans les événements qui les entourent faisant d'eux de meilleurs citoyens (Gilmor, 2006, p. 18). Pour Aubert, il relèverait d'un «processus actif d'auto-définition » (Aubert, 2014, p. 127).

C'est aux côtés de ces multiples champs et interrogations que s'inscrit la contribution de la Caravane des médias d'initiative populaire. Avec ceci de particulier qu'elle n'étudie ni le militantisme collectif, ni la collaboration pro-am, mais la volonté individuelle de "faire média » via internet.

\section{La Caravane des médias d'initiative populaire}

La «Caravane des médias d'initiative populaire », qui s'insère dans un programme de recherche plus vaste qui étudie l'espace démocratique et la citoyenneté ${ }^{1}$, est partie scientifiquement et matériellement à la recherche des formes alternatives et participatives des nouvelles sociabilités citoyennes. Nous avons recherché quels médias étaient créés en ligne par initiative populaire sur une zone délimitée du sudouest de la France, incluant à la fois des territoires urbains et ruraux, sur cinq départements de la région Aquitaine ${ }^{2}$. L'objet recherché a été défini, a priori, comme un média ayant été créé sur Internet du fait de l'action d'un individu ou de plusieurs individus animés par la volonté de « faire média » ou de « faire débat » mais n'étant pas des professionnels (du journalisme, de la politique ou d'une entreprise).

Cet objet d'étude, disons le ouvertement, est complexe à appréhender tant aussi bien du point de vue de la variété des formes prises par ces médias en ligne que par la diversité des intentions et

1 Le programme Citoyenneté en couleurs, co-dirigé par Alain Bouldoires et Christine Larrazet, inclue trois terrains de recherche sur la prise de parole dans l'espace public (La caravane des médias d'initiative populaire, Le Blasphème en Europe, Le Slam) et un axe de réflexion méthodologique sur les méthodes visuelles en sciences sociales.

2 Au moment où nous avons conduit l'étude, avant la réforme des régions françaises, la région Aquitaine comprenait cinq départements : Dordogne, Gironde, Landes, Pyrénées Atlantiques, Lot et Garonne. 
des lignes éditoriales plus ou moins explicitées. D'une première phase d'inventaire ${ }^{1}$, volontairement non exhaustive, ont émergé près d'une centaine de pistes dans les trois types de médias sélectionnés : webradio, webTV et webmagazine. Cette phase exploratoire a été suivie par une enquête par observation participante - une cyberanthropologie des médias - afin d'affiner la recherche et d'écarter les médias qui ne correspondaient pas aux critères de l'étude. Ont été ainsi retirés les médias dont il s'avérait qu'ils étaient adossés à un média déjà existant, qu'il soit mainstream ou alternatif, préalablement diffusé sur papier ou sur la FM. Ont également été écartées les créations en ligne adossées à un groupe organisé dont elles faisaient office de porte-parole, que ce soit une structure associative ou institutionnelle, et dont l'intention était de s'adresser uniquement à des adhérents. Le média devait être une création online ex nihilo par des " acteurs-moteur amateurs " voulant « faire média ou débat » au-delà de leurs soutiens ou adhérents.

La cyberanthropologie des médias a, d'autre part, permis de pénétrer le monde des pratiques médiatiques virtuelles et de mieux comprendre, de l'intérieur, les moyens et les ressorts de l'engagement citoyen. Ce travail d'immersion était nécessaire afin de mieux identifier les acteurs sources et de commencer à entrer en contact avec eux, notre intention finale étant, dans un troisième temps, de conduire un entretien semi-directif avec ces citoyens média-actifs sur leur engagement et leur prise de parole dans l'espace public. A ce troisième stade, un paradoxe est apparu. Ces citoyens qui pénètrent de leur propre fait l'espace public étaient extrêmement difficiles à joindre, et plutôt réticents à être « saisis » dans une étude ${ }^{2}$. Au final, 20 entretiens ont pu être conduits.

Tableau des entretiens effectués par département et type de média

\begin{tabular}{|c|c|c|c|}
\hline & Web Presse & Web Radio & Web TV \\
\hline GIRONDE & 3 & 1 & 2 \\
\hline PYRENNEES & 3 & 1 & 4 \\
ATLANTIQUES & & & \\
\hline DORDOGNE & 2 & 1 & 0 \\
\hline LANDES & 1 & 1 & 1 \\
\hline LOT ET GARONNE & 0 & 0 & 0 \\
\hline
\end{tabular}

1 https://www.msha.fr/caravanedesmedias/

2 https://www.msha.fr/caravanedesmedias/ 
De ces entretiens ont été sélectionnés 8 figures auxquelles nous avons consacré des portraits audio-visuels (diaporama sonore ou audio slideshow) au cours d'une itinérance sur les routes d'Aquitaine avec un studio caravane (Wunderstudio ${ }^{l}$ ). Par ces portraits, consultables en ligne sur un site dédié ${ }^{2}$, ont été mises en pratiques les méthodes visuelles communes à tous les axes du programme Citoyenneté en couleurs pour la restitution et le partage de l'étude hors champ scientifique.

Notre intention première et finale n'était pas la caractérisation sociologique de ces citoyens « média-actifs » bien que ce volet soit un possible développement de l'étude. La visée de la Caravane des médias d'initiative populaire, allant à la rencontre de citoyens producteurs de contenus informationnels, n'était pas davantage de contribuer au débat opposant les thèses de la mobilisation et du renforcement, qui cherche à déterminer si ces citoyens qui font débat en ligne sont distincts ou bien sont les mêmes que ceux déjà engagés politiquement hors ligne, notamment en termes d'âge, de catégorie socioprofessionnelle et de genre (Oser, Hooghe, Marien, 2013).

Dans notre corpus cohabitent un ensemble de médias hétéroclites qui ne semblent pas partager de traits communs, à l'exception du critère central de sélection qui requiert que le média en ligne ait été créé ex nihilo par initiative populaire sans accompagnement professionnel. L'objet trouvé est multiple et il semble mieux définissable par ce qu'il n'est pas, ou ce qu'il n'est pas systématiquement. Ainsi, ces médias ne sont pas tous alternatifs et ne se placent pas, si l'on suit la définition de Cardon et Granjon, en contrepoint aux médias dominants dans une volonté anti-hégémonique (Cardon \& Granjon, 2010). Ils ne sont pas tous militants dans le sens où ils seraient un soutien informationnel $\grave{a}$ une cause commune. Et tous ne sont pas des weblogs individualisés. En revanche, tous ont volonté d'être partie prenante d'un processus démocratique.

Créés entre 2007 et 2012, les médias que nous avons découverts ont pris corps dans une pluralité d'intentions : valoriser et pratiquer une langue locale, soutenir un candidat à l'élection présidentielle, tisser des liens avec des personnes isolées dans un quartier urbain en déshérence, partager des images photographiques d'un territoire commun, surveiller et commenter la politique locale, militer pour la reconnaissance d'une

$1 \mathrm{http}: / /$ wunderstudio.fr/

$2 \mathrm{http}: / /$ www.msha.fr/caravanedesmedias/ 
identité territoriale. Ces intentions diverses partagent cependant un trait commun qui fait leur unité : la volonté première qui a présidé à la création de ces médias en ligne est d'occuper un espace territorial laissé vacant.

\section{Réinventer la citoyenneté}

Selon l'enquête d'Harris Interactive réalisée du 25 au 27 janvier 2016 auprès de 1624 personnes, $53 \%$ des français affirment se sentir engagés dans la vie de la société. Mais paradoxalement cet engagement citoyen se manifeste principalement par des actions individuelles (le vote, le tri des déchets, s'occuper d'un proche, donner du sang, avoir une consommation responsable...). Une enquête plus ancienne montrait pourtant qu'un quart des français sont bénévoles au sein d'une association ( $28 \%$ des personnes de plus de 15 ans - enquête «Vie associative» de l'Insee, 2002). De manière assez inattendue, ce qui mobilise relève plutôt des domaines de la culture, des loisirs et de la défense des droits que de l'action sociale ou des œuvres caritatives. Les auteurs de cette étude, Lionel Prouteau et François-Charles Wolff, observent la présence de «deux complexes de motifs assez distincts, l'un tourné vers l'attention à l'ego, l'autre orienté davantage vers le souci d'autrui » (Prouteau, Wolff, 2004, p. 29). Les motifs de l'engagement sont donc complexes : philanthropie, valeurs politiques, débouché professionnel, estime de soi, recherche identitaire, éthique, plaisir... Les associations participent donc, à leur manière, à la richesse d'une nation entre individualisation et participation. Il s'agit pour chacun de «fonder le sens de ses propres engagements 》 (Vermeersch, 2004, p. 689). Autre chiffre marquant : plus de $50 \%$ des responsables associatifs sont des retraités. Aujourd'hui le retraité innove et représente une force active et citoyenne d'engagement (Guillemard, 2002, p. 53). Plus globalement, nous assistons à un renouvellement des formes d'engagement qui s'affranchit des appartenances et des affiliations partisanes ou sociales. L'espace public, en tant que sphère politique, permet de sortir de la masse ou de l'entre-soi, de parler en nom propre sur des enjeux locaux, de faire se rencontrer des mondes sociaux différents.

Notre enquête menée auprès de citoyens média-actifs s'intéresse à une des formes de cette nouvelle donne de l'engagement pluriel. Elle montre, loin de la naïveté dont on les accuse souvent, comment les médias qu'ils ont conçus en ligne sont devenus de véritables laboratoires du débat public. Face au journalisme de révérence ou de connivence 
des médias traditionnels dénoncé par Serge Halimi (Halimi, 1997 ; Halimi \& Larrazet, 2012), ces initiatives constituent un foisonnement de prises de parole qui expérimentent un renouvellement du rapport à l'information et à la chose politique. En effet, ces citoyens, qui ne se reconnaissent pas dans les pratiques habituelles du débat démocratique, n'ont rien perdu de leur engagement : ils puisent dans leur réalité locale la volonté de partage qui mobilise, pour l'essentiel, leur énergie.

Les acteurs que nous avons trouvés ne sont pas véritablement des «médiactivistes », au sens de Cardon et Granjon (Cardon\& Granjon, 2010) ${ }^{1}$, qui partageraient, comme motivation fondamentale et fondatrice, la volonté de s'opposer ou de fournir un contrepoint aux médias d'information traditionnels. Et ils ne se définissent pas eux-mêmes selon les deux orientations que distinguent Cardon et Granjon parmi les médiactivistes, l'orientation anti-hégémonique ou expressiviste. Pour expliquer la naissance de leur action créative, ils soulignent tous la volonté d'occuper une vacance laissée par les médias d'informations. Cette vacance est définie premièrement en termes géographiques et seulement, en second lieu, comme une non prise en compte de paroles et de voix issues de leur territoire.

«Ce qui nous différencie des médias dominants, c'est qu'on considère le média comme un lien social, dans la proximité avec des personnes qui vivent sur un même territoire. Pour nous aujourd'hui, c'est cela qui est absent des médias dominants vu qu'ils sont sur des modèles de territoires trop larges, qui font que cette proximité n'existe plus.»

Ximun Carrère, Kanaldude (WebTV).

« On a envie de parler de ce qui se passe sur le territoire, mais on n'est pas des journalistes et je pense que ce qu'on sait bien faire c'est mettre en avant ce qui se passe de bien. [...] Il y a tellement de choses positives qui se passent sur le territoire que c'est là que se situe mon engagement. » Franck Zordan, Ici Tele (WebTV).

1 Dominique Cardon et Fabien Granjon définissent l'univers du médiactivisme comme «les mobilisations sociales progressistes qui orientent leur action collective vers la critique des médias dominants et/ou la mise en cuvre de dispositifs alternatifs de production d'information », page 8 . 
" C'est assez marrant à expliquer mais je suis fils de commerçant et j'ai un attachement profond, je suis très sensible, aux valeurs de travail et de production de choses locales, pour le quotidien, pour l'animation d'un territoire, pour la proximité des gens. [...]. Je me suis dit que je pouvais peut-être essayer de créer quelque chose qui me permettrait d'une part, de valoriser ce que j'ai connu et toutes les valeurs auxquelles je suis attaché, et qui me permettraient de donner un sens local à Internet. [...] Je crois surtout qu'Internet est là également pour favoriser la proximité locale. Tout ça, pour moi, c'est fondamental. » Gérard Fauveau, WebtvPau (WebTV)

Les créateurs de ces médias d'initiative populaire, sont davantage des média-acteurs de proximité que des "médiactivistes ", un terme dans lequel aucun de ceux que nous avons interrogé ne s'est reconnu et que certains ont fermement repoussé en argumentant qu'ils n'étaient pas des " activistes ». En passant par l'espace public numérique, ils agissent dans et sur un territoire bien délimité comme " acteurs de proximité » et diffuseurs d'information. Ce champ de pratiques, à la fois profondément ancré dans un territoire et techniquement déterritorialisé, est à l'image d'un monde contemporain où s'articulent le global et le local. La recherche de l'action, le goût du concret s'expriment à travers un pragmatisme de la parole comme méthode d'intervention.

\section{Réincarner le lien social}

Entre 2012 et 2015, nous avons ainsi enquêté sur un champ pluriel où la parole est assimilée, le plus souvent, à une action, ce que John Langshaw Austin qualifiait d'énoncés performatifs (Austin, 1970). Le langage est ici un instrument d'action, la prise de parole est un acte qui n'a pas seulement pour fonction de dire mais aussi de provoquer des effets réels. Pour la plupart de ces citoyens média-acteurs, une expression n'est pas une simple énonciation. Elle trouve son sens dans une intention qui est en relation avec des faits. Son but principal est opérationnel. Joindre le geste à la parole est l'idéal type d'une vraie culture politique qui franchit le pas d'une pensée intérieure qui devient publique et recoupe le concept de vita activa défini par Hannah Arendt. Cette parole est tout simplement le signe d'un engagement, certes avec des effets qui ne sont pas nécessairement immédiats (c'est-à-dire 
performatifs au sens strict d'Austin) mais qui visent une intervention citoyenne dans les affaires humaines.

« J'avais cette envie de partager quelque chose de fort, d'échanger avec les gens. De participer et de faire participer. D'où le nom du blog 'Aux Pensées Citoyens !', en réaction à 'Aux Armes Citoyens' que je trouvais trop violent. 'Aux Pensées' fait écho à ma personnalité, puisque je me considère comme un passeur de mots. Je suis persuadée que les mots ont des pouvoirs puissants, et qu'ils peuvent faire bouger des montagnes. Grâce aux mots, on peut faire très mal, on peut blesser, mais on peut aussi faire avancer les choses. »

Marie-Christine Darmian, Aux Pensées Citoyens (Webmagazine).

Leur prise de parole ne rend pas seulement compte, elle participe également aux faits en posant des mots. Faisant acte de témoignage, le média-acteur agit sur la scène sociale en s'appuyant sur des valeurs. Il envisage les conséquences de son acte qui ne sont plus de l'ordre du dire : le langage pèse son poids sur la vie ordinaire, tel est le pari. Le phénomène relève de l'engagement, de l'intention, du comportement social à distinguer donc de la pratique journalistique habituelle. On voit toute la difficulté à saisir un objet à étudier qui échappe aux catégories habituelles : informer ou exécuter un acte se rejoignent dans un même objectif citoyen.

Notre enquête révèle que les citoyens média-actifs utilisent Internet pour agir sur un monde commun et, également, qu'ils articulent leur espace informationnel avec la matérialité du « monde réel ». C'est ce que montre notamment le témoignage de Françoise Boulanger, auteure du blog Le canard à l'orange, que nous avons rencontré. Membre du Modem (parti politique centriste), son activité de bloggeuse a prolongé son engagement politique et l'a, peut être même, intensifié. Son activisme numérique est complémentaire à son travail de terrain car elle entend influencer le «monde réel». Son blog est une tribune au service de l'efficacité et de la réflexion. Pour Françoise Boulanger, le blog est surtout un prétexte pour exposer ses idées et tenter de mettre en place un certain nombre de projets issus d'une pratique consciente de la sérendipité. Elle s'est créée un personnage, la « dame en rouge », 
aisément identifiable et connue dans sa ville de Dax. Son action citoyenne n'est donc pas que virtuelle, elle est aussi matérielle et incarnée.

La prise de parole est également une prise de risque. Parce que les média-acteurs de proximité s'expriment dans un espace géographique délimité, qu'ils l'investissent et qu'ils s'y engagent, plusieurs créateurs de média en ligne ont mesuré le poids de leur parole. Des retours physiques et verbaux menaçants sont venus donner corps à cette prise de risque. Ainsi le créateur du site Adiu, dans une petite ville de Gironde, a reçu plusieurs coups de chevrotine sur son véhicule portant la marque de son site (voir entretien en ligne ${ }^{1}$ ). Ainsi Marie-Christine Darmian, créatrice d'un weblog politique sur le bassin d'Arcachon, a-telle mesuré les conséquences de la prise de parole :

« Il y a eu des moments où j'ai dit « j'arrête tout ». Voilà. « J'arrête tout », parce que la connerie humaine, je n'étais pas capable de l'appréhender. La méchanceté aussi. A une époque, je recevais une lettre anonyme par jour. C'est épuisant. C'est épuisant. »

Marie-Christine Darmian, Aux Pensées Citoyens (Webmagazine).

\section{Produire le commun}

La question n'est pas tant de savoir si le média-acteur de proximité a raison, si ses affirmations sont opportunes ou si c'est bien ce qu'il convenait de dire, mais de savoir s'il est légitime pour un citoyen de s'impliquer de cette manière dans les affaires publiques. Internet a été appréhendé de façon très optimiste comme un outil pouvant activer la démocratie. Pour Olivier Blondeau, l'enjeu n'est pas tant que ce dispositif puisse ou non «réenchanter la démocratie » il est davantage « d'élaborer à travers la technique [...] des manières et des formes d'agir ensemble, de produire du commun en tenant compte de la singularité de chacun. »(Blondeau, 2007, p. 372 et p.380). Ici se situe l'enjeu des médias de proximité. Les média-acteurs ne sont pas des internautes en chambre, des activistes en fauteuil ou en chaise longue. Ils descendent dans la rue, rencontrent les citoyens, interpellent leur ville ou leur village. Grâce aux technologies de mobilité, certains produisent directement sur place, là où la curiosité les dirige. A travers ces liens

$1 \mathrm{http} / /$ www.msha.fr/caravanedesmedias/ 
tissés au fil des échanges, des espaces de libre expression s'ouvrent et rendent concrètes des expérimentations citoyennes incarnées dans une culture du partage retrouvée. Ainsi, la webradio Toobordo fait circuler son « bâton de parole » entre jeunes et vieux pour mettre en commun ce qui ne l'est pas ou ne l'est plus (voir entretien en ligne').

Au-delà des fascinantes fonctionnalités de l'outil, nous serions face, selon Laurence Allard, à l'émergence de nouvelles cultures expressives issues d'un écosystème numérisé et d'une révolution du « pouvoir-dire » (Allard, 2007, p.61). Entre production personnelle et production à vocation professionnelle, notamment du côté des webTV dont le coût demande une obligatoire rentabilité, le champ occupé par les média-acteurs de proximité est large et traduit des engagements différenciés mais que l'on peut assimiler à des pratiques sociales. En effet, cette culture du flux est avant tout une culture de l'échange hypersocialisante. Le média d'initiative populaire se présente comme l'architecture de nouvelles formes sociales produisant du commun grâce à un travail expressif ordinaire. Ainsi le co-créateur et coanimateur de Kanaldude, une webTV conçue et diffusée uniquement en langue basque dans un village au pied des Aldudes, cherche avant tout à rassembler, faire communiquer, et faire « dire » les habitants d'un territoire dont il a vu qu'ils n'échangeaient plus, comme ils le faisaient autrefois au bar à la sortie de l'église (voir entretien en ligne ${ }^{2}$ ). Au lieu d'appliquer un regard extérieur et surplombant, Kanaldude fait le choix, avant la réalisation d'un sujet, de réunir les personnes impliquées dans ce sujet, parfois dans les locaux de Kanaldude, afin de participer à la conception audioviduelle par la mise en parole.

« Pendant des années cette proximité a été un peu décriée, ou sous valorisée, en disant que c'était une télé miroir, que de toute façon les gens, ce qui les intéressaient, c'était de se voir, que ce n'était pas intéressant et que ça ne les tiraient pas en avant. Nous, on revient de cette réflexion là. Par l'action qu'on fait, on montre que ça se passe différemment. Dans la phase de réflexion, entre une idée et sa réalisation audiovisuelle, il y a là un temps d'échange qui est très important pour mieux connaître son territoire, mieux connaitre son voisin, discuter... Et c'est ça, pour

1 http://www.msha.fr/caravanedesmedias/

$2 \mathrm{http} / / /$ www.msha.fr/caravanedesmedias/ 
nous, ce qui nous différencie, et c'est ça qu'on pose sous l'aspect participatif. »

Ximun Carrère, Kanaldude (WebTV).

\section{Conclusion}

La sociologie des médias numériques a, dès les années 1990, cherché à évaluer si l'espace numérique serait un espace en rupture avec l'espace « réels » des interactions. Y-a-t-il rupture entre les mondes offline et online, ou bien continuité ? Les codes et les normes présidant à l'interaction seraient-elles aménagées ?

Concernant les média-acteurs de proximité que nous avons rencontrés, il n'y a nullement un " adieu au corps » prophétisé par David Le Breton (Le Breton, 1999), c'est-à-dire une dématérialisation de l'être, mais bien au contraire un réinvestissement de la vie publique par le numérique, une incarnation de la citoyenneté. A. Casilli explique que « la propagation de représentations corporelles en ligne atteste des désirs et des attentes que les usagers projettent sur leur propre corps » (Casilli, 2010, p. 328). Le corps des média-acteurs de proximité n'est pas virtuel ou idéalisé, il est transformé, dans toute sa matérialité, par le lien social dont ils font un usage quasi professionnel. Les rencontres directes, les relations aux autres, les multiples contacts en face-à-face font partie intégrante de leurs pratiques numériques. Entre le média et le terrain, les échanges se poursuivent dans un sens ou dans l'autre. Les liens numériques ont leurs spécificités : ils ne concernent pas l'amitié mais une nouvelle modalité de la relation sociale qui rompt avec le cadre social habituel; des liens que l'on peut qualifier le plus souvent de faibles mais qui peuvent aussi se révéler des liens forts en rapport avec une appartenance commune ou un intérêt commun. Au fond, les médias d'initiative populaire, dignes héritiers de la presse révolutionnaire, du cinéma militant ouvrier, des radios libres et des médias communautaires, nourrissent de nouvelles relations sociales, inventent de nouveaux liens et dynamisent la citoyenneté.

Le web 2.0, en tant que laboratoire social travaillant l'identité territoriale, participe d'une massification des processus de subjectivation. La " prise en main » par le plus grand nombre des techniques de l'Internet renverse, de manière inédite, un certain ordre culturel et informationnel établi. Les pratiques éditoriales, entre exploration personnelle et sociale, remanient profondément le sens du collectif. Car le média-acteur de proximité, auteur de formes social- 
identitaires territoriales, expérimente la narration d'un nous à partir de sa subjectivité et de son engagement sur le terrain.

Même si parfois ils peuvent prendre la forme de « journaux extimes» (Flichy, 2010), dans le cas des weblogs politiques par exemple, ces pratiques, le plus souvent, donnent à voir de nouveaux horizons. Loin d'un repli sur soi, la production de ces citoyens média-actifs révèle une polyphonie du sujet conçue dans le dialogue. Ainsi s'articule « technologie du soi » (Bouldoires, Vacaflor, 2009) et souci de l'autre, identité personnelle et collective, différentes facettes de l'individu et du social. Dans cette pratique du lien, on perçoit un paysage mouvant et subjectif, fait de multiples singularités qui s'agrègent autour d'intérêts communs. Cette interconnexion a un effet structurant certain, sans pour autant aboutir véritablement à des mobilisations, les mouvements sociaux et culturels utilisant d'autres architectures sociotechniques. C'est à l'échelle du territoire que s'agrègent les individus et que les médias d'initiative populaire expérimentent une énonciation collective et singulière.

«Si la passion démocratique semble aujourd'hui faire défaut, ce n'est pas faute de volonté citoyenne mais faute d'oxygène, faute d'espace, faute de temps pour l'imagination créative des citoyens [...]. Il est vrai qu'une telle application radicale de la démocratie révolutionne pas mal de pouvoirs établis. " conclut le sociologue Antoine Bevort (Bevort, 2002) dans son ouvrage qui analyse les apports des délibérations locales au renforcement du « capital social» et à la vitalité de la démocratie. Plus que jamais, la prise de parole représente un engagement à haut risque, l'horizontalité des échanges dérangeant la verticalité des rapports sociaux institués. Ainsi, au moins trois catégories de conservatismes locaux sont confrontées à la libération de l'expression citoyenne : les féodalités politiques, les routines journalistiques, les préjugés individuels.

\section{Références}

Allard, L. (2007). Emergence des cultures expressives, d'internet au mobile. Médiamorphoses, (21), 19-25 et 57-62.

Arendt, H. (2012). Condition de l'homme moderne. Dans H. Arendt, L'Humaine condition, coll. Quarto. Paris : Gallimard.

Aubert, A. (2009). La société civile et ses médias. Quand le public prend la parole. coll. I.N.A. Paris: Le Bord de l'eau.

Austin, J. L. (1970). Quand dire, c'est faire. Paris : Éditions du Seuil. 
Badiou, A., \& Gauchet M. (2014). Que faire? Paris : Philosophie Editions.

Bevort, A. (2002). Pour une démocratie participative. Paris : Presses de Sciences po. Blondeau, O. (2007). Devenir média. Paris : Editions Amsterdam.

Bouldoires, A., \& Vacaflor, N. (2009). Repères identitaires et médiations technologiques : de nouveaux espaces relationnels dans les ghettos. Dans F. Dervin \& Y. Abbas (Éd.), Technologies numériques du soi et (co-) construction identitaires, Questions contemporaines. (pp.83-103). Paris : L'Harmattan.

Calhoun, C. (2010). The Public Sphere in the Field of Power. Social Science History, (34:3), 301-335.

Cardon, D., \& Granjon, F. (2010). Médiactivistes, coll. Contester. Paris : Presses de Sciences Po.

Cardon, D., \& Smoreda Z. (2014). Réseaux et les mutations de la sociabilité. Réseaux, (184-185), 161-185.

Casilli, A. (2010). Les liaisons numériques, coll. La couleur des idées. Paris : Éditions du Seuil.

Denouël, J., Granjon F., \& Aubert A. (2014). Médias numériques et participation, coll. MediaCritic. Paris: Mare \& Martin.

Flichy, P. (2010). Le sacre de l'amateur, coll. La République des idées. Paris : Éditions du Seuil.

François, B., \& Neveu, E., (1999) (dir.). Espaces publics mosaïques. Acteurs, arènes et rhétoriques des débats publics contemporains. Rennes : Presses Universitaires de Rennes.

Fraser, N. (1990). Rethinking the Public Sphere: A Contribution to the Critique of Actually Existing Democracy. Social Text, (25/26), 56-80.

Gillmor, D. (2006). We the Media. Grassroots Journalism by the People, for the People. Sebastopol: O'Reilly Media.

Gingras, A. (2009). Médias et démocratie. Le grand malentendu. Québec : Presses de l'Université du Québec.

Grossetti, M. (2014). Que font les réseaux sociaux aux réseaux sociaux ? Réseaux, (184-185), 187-209.

Guillemard, A-M. (2002). De la retraite mort sociale à la retraite solidaire. Gérontologie et Société, (102). Disponible à : https://www.cairn.info/revue-gerontologie-etsociete1-2002-3.htm

Habermas, J. (1988). L'espace public. Archéologie de la publicité comme dimension constitutive de la société bourgeoise. Paris : Payot.

Habermas, J. (1992). «L'espace public», 30 ans après. Quaderni, (18), 161-191.

Halimi, S. (1997). Les nouveaux chiens de garde. Paris : Raisons d'agir.

Halimi, S., \& Larrazet, C. (2012). At the Crossroads of Media, Media Critique, and the Critique of Media critics - An Interview with Serge Halimi, Editor-in-Chief of Le Monde Diplomatique. InMedia (2), Disponible à: http://inmedia.revues.org/500.

Le Breton, D. (1999). L'adieu au corps. Paris : Métaillé.

Oser, J., Hooghe, M., \& Marien S. (2013). Is Online Participation Distinct from Offline Participation? A Latent Class Analysis of Participation Types and Stratification. Political Research Quarterly, (66:1), 91-101.

Pélissier, N., \& Chaudy, S. (2009). Le journalisme participatif et citoyen sur Internet : un populisme dans l'air du temps ?. Quaderni (70). Disponible à : http://quaderni. revues.org $/ 512$

Perret, B. (2003). De la société comme monde commun. Paris : Desclée de Brouwer. 
Prouteau, L., \& Wolff, F-C. (2004). Donner son temps : les bénévoles dans la vie associative. Economie et statistiques, (372). Disponible à : http://www.insee.fr/fr/ themes/document.asp?reg_id $=0 \&$ ref_id $=$ es $372 \mathrm{a}$

Putnam, R. (2000). Bowling Alone. The collapse and revival of American community. New York : Simon \& Schuster.

Rodríguez, C. (2001). Fissures in the Mediascape. An International Study of Citizens' Media. Cresskill, NJ: Hampton Press.

Tassin, E. (1999). Le trésor perdu. Hannah Arendt, l'intelligence de l'action politique. Paris : Payot.

Vermeersch, S. (2004). Entre individualisation et participation : l'engagement associatif bénévole. Revue Française de Sociologie, (Vol. 45/4). Disponible à : https://www. cairn.info/revue-francaise-de-sociologie-2004-4.htm 